\title{
A RELIABLE AND ENERGY EFFICIENCT ROUTING PROTOCOL FOR MANETS
}

\author{
Pallavi Kaliyar ${ }^{1}$, Kavita Pandey ${ }^{2}$, Gagan Singla ${ }^{3}$ \\ ${ }^{1}$ Department of Computer Engineering, Shri Ram College of Engineering \& \\ Technology, Muzaffarnagar, India \\ pallavi.kaliyar@gmail.com \\ ${ }^{2}$ Department of Computer Engineering, Jaypee institute of information \\ technology, NOIDA, India \\ kavita.pandeyejiit.ac.in \\ ${ }^{3}$ Department of Computer Engineering, Aryabhatta Group of institutions, \\ Barnala, India \\ vipulgog@gmail.com
}

\begin{abstract}
A mobile ad-hoc network (MANETs) is an infrastructure less network in which the mobile nodes communicate with each other. Due to its various characteristics like highly dynamic topology and limited battery power of the nodes, routing is one of the key issue. Also, it is not possible to give a significant amount of power to the mobile nodes of ad-hoc networks. Because of all this the energy consumption is also an important issue. Due to limited battery power, some other issues like if some node gets fail, which results in loss of data packets and no reliable data transfer has been raised. In this paper, an algorithm is proposed for data transmission which detects the node failure (due to energy) before it actually happens. Because of this network lifetime gets improved. The proposed routing algorithm is energy efficient as compared to $A O D V$ routing algorithm. The performance is analyzed on the basis of various performance metrics like Energy Consumption, Packet Delivery Ratio, Network Life Time, Network Routing Overhead and number of Exhausted nodes in the network by using the NS2 Simulator.
\end{abstract}

\section{KEYWORDS}

Network Protocols, Wireless Network, Mobile Network, Energy Efficiency, AODV \& Rfactor.

\section{INTRODUCTION}

MANETs are collection of mobile hosts (also called nodes) which are self-configure-able, selforganize-able and self-maintain-able. Unlike the cellular network which is a single hop. In Mobile ad-hoc networks (MANETs) mobile devices connected to each other by dynamic topology and a multi-hop approach without having any central access point. In ad-hoc network if a device wants to communicate with any other device it is not necessary that both nodes should be in each other transmission range. They can be anywhere in the network and still communicate through other

Sundarapandian et al. (Eds): CoNeCo,WiMo, NLP, CRYPSIS, ICAIT, ICDIP, ITCSE, CS \& IT 07, pp. 225-235, 2012. (C) CS \& IT-CSCP 2012

DOI : $10.5121 /$ csit.2012.2419 
mobile devices. It is very useful in those areas where cellular communication is not feasible or affordable.

In MANETs one of the most important issue is routing i.e. finding a suitable route or path from source to destination. As these are mobile devices which are not fixed, so these devices have limited life. As the energy is consumed even when the device is in idle state or not in the real data transfer pathway just by receiving route request packets. When the battery drains out they became dead, which results in inability to send or receive packets. So, it will result not even in its own data loss but also it leads to whole network failure by not sending others devices data packets. A lot of research has been done to reduce this energy consumption in ad-hoc networks so that the network lifetime can be improved.

The energy efficient routing protocols [23] [10] must be designed by keeping a view on nodes and the network. The network lifetime is directly influenced by the nodes lifetime because the nodes act in the network as router and host. One way to reduce the energy consumption is by selecting the route with less energy consumption for end-to-end packet transmission. However these kinds of routing protocols save significant amount of energy, but they don't guarantee to prolong the network lifetime. Sometimes these protocols may shorten the network lifetime since they use specific routes repeatedly by considering the end-to-end energy consumption. Here our aim is to reduce this extra energy consumption in a network so as to make the network life as long as possible. To achieve this, we designed energy efficient routing protocol for MANETs which discovers the route based on energy aware metrics, bypasses the poor-energy nodes and at the same time pick a route that will consume less energy.

This paper is divided into a number of sections. In section 2 , the related work done by various researchers has been presented. Proposed algorithm is given in section 3. In section 4, simulation parameters and simulation results has been given. This is followed by conclusion in section number 5 .

\section{RELATED WORK}

In MANETs battery power is limited, the efficient battery power management is essential to increase the lifetime. Several researches have worked in this direction and their works is as follows:-

Sandip Vijay et al. [3] proposed an approach named as Energy Efficient Network (EEN) which elects "controllers, backbones and connectors" from the given network. EEN backbones stay awake continuously and perform multi-hop packet and switching routing while other nodes in the network remain in power-saving mode and periodically check whether they can be backbone node or not. Author checked the energy efficiency of their algorithm on the basis of network lifetime. Duy Ngoc Pham et al. [12] proposed another technique named as Expanding Ring Search (ERS) this is used to reduce the number of RREQ packets, so that the nodes only process necessary packets and discard duplicate packets to reduce overheads and to use energy efficiently by using the Time to Live (TTL) mechanism. Author proves their result on the basis of reduced overhead and energy consumption in the network. After this an improved ERS scheme is proposed by Preethi et al. [5] named as Energy Efficient Expanding Ring Search Technique $(E E A O D V)$. This approach is applied to the route discovery phase and saves energy of the nodes by avoiding the redundant rebroadcasting of the RREQ packets. The relaying status of the node is 
decided based on the broadcasting of the RREQ packets by its neighbors. This technique reduces routing overheads incurred during the route discovery process and decreases the energy consumption even more as compared to the ERS approach.

Ashwani Kush et al. [4] proposed an approach named as COMMON POWER which works to find the minimal common value of node transmission range to maintain the network connectivity by choosing the nodes of same battery power. COMMON POWER attempts to satisfy three major objectives increase the battery lifetime of all the nodes, increase the traffic carrying capacity of the network and reducing the contention among the nodes. Authors validate their proposed protocol by calculating number of exhausted nodes. Kwan-Woong Kim et al. [7] comes up with another approach named as Local Route Change Algorithm (LRCA). The objective of this approach is to reduce route failure caused by dead nodes which consume all the battery life. This technique provides an ability of changing routes to neighboring nodes from a node which has low battery and focus on route discovery to minimize power consumption or effective power distribution before some of intermediate nodes fail due to their battery. Author proved their results by showing a decrement in the number of lost packets.

LI Bing et al. [11] comes up with Cross-Layer Design of Energy approach which adopts crosslayer mechanism and energy-aware metric to improve routing protocol to reduce the energy consumption and then prolong the life of the whole network. In this approach, the link layer and the routing layer work together to choose the optimized transmission power for nodes and the route for packets. The link layer provides the energy consumption information for the routing layer and the routing layer chooses route accordingly and conversely controls the link layer to adjust the transmission power. They have analyzed their approach on the basis of energy consumption and average delay. Author proved the performance is better when the traffic load is higher in the network and reduce the energy consumption by $8 \%$ of the network. After this Sunil Taneja et al. [13] recommended an algorithm named as Power Management of the route. This approach takes the consideration of power awareness during route selection. They observe power status of each and every node in the topology and further ensure the fast selection of routes with minimal efforts and faster recovery. The scheme is incorporated with the AODV protocol and the performance has been studied through simulation which shows as a result of less number of dead nodes in the network and author validate their proposed algorithm by using packet delivery ratio and number of exhausted nodes in the network.

Comparison of above described work on the basis of base protocol, performance metrics etc. is given in the table below. 
Table 1: Related Work

\begin{tabular}{|c|c|c|c|c|}
\hline $\begin{array}{l}\text { Author } \\
\text { Name } \\
\text { Reference }\end{array}$ & $\begin{array}{l}\text { Base } \\
\text { Protocol }\end{array}$ & Simulator & Performance Metrics & $\begin{array}{l}\text { Variable } \\
\text { parameters }\end{array}$ \\
\hline $\begin{array}{l}\text { Sandip Vijay } \\
\text { et al.[3] }\end{array}$ & DSR & $\begin{array}{l}\text { NS2[18] \& } \\
\text { OPNET }\end{array}$ & $\begin{array}{l}\text { Packet delivery rate, } \\
\text { Coordinators } \begin{array}{r}\text { density, } \\
\text { Cumulative distribution } \\
\text { per link }\end{array}\end{array}$ & $\begin{array}{l}\text { Pause time, Node } \\
\text { density, Delivery } \\
\text { rates }\end{array}$ \\
\hline $\begin{array}{l}\text { Ashwani } \\
\text { Kush et al.[4] }\end{array}$ & $\begin{array}{l}\text { AODV } \\
\& \text { DSR }\end{array}$ & NS2[18] & $\begin{array}{l}\text { Exhausted nodes, Total } \\
\text { energy consume }\end{array}$ & No of Sources \\
\hline $\begin{array}{l}\text { S.Preethi et } \\
\text { al.[5] }\end{array}$ & AODV & $\begin{array}{l}\text { GloMoSim } \\
{[16]}\end{array}$ & $\begin{array}{l}\text { Energy } \text { Consumption in } \\
\text { sparse and dense } \\
\text { medium. }\end{array}$ & $\begin{array}{l}\text { No of Node, No of } \\
\text { Speed }\end{array}$ \\
\hline $\begin{array}{l}\text { Kwan-Woong } \\
\text { Kim et al.[7] }\end{array}$ & AODV & NS2[18] & No of packets. & $\begin{array}{l}\text { No of } \text { CBR } \\
\text { Connections }\end{array}$ \\
\hline $\begin{array}{l}\text { Nivedita } \mathrm{N} . \\
\text { Joshi et al.[9] }\end{array}$ & LAR & QualNet & 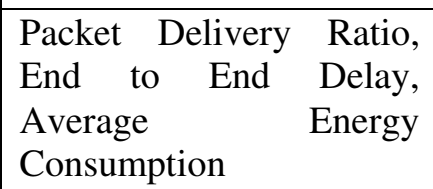 & $\begin{array}{l}\text { No of Nodes, No } \\
\text { of Speed, Packet } \\
\text { Rate. }\end{array}$ \\
\hline $\begin{array}{l}\text { LI Bing et } \\
\text { al.[11] }\end{array}$ & AODV & $\mathrm{NS} 2[18]$ & $\begin{array}{l}\text { Average Delay, } \\
\text { Consumed Energy. }\end{array}$ & $\begin{array}{l}\text { No of Nodes, No } \\
\text { of Connections. }\end{array}$ \\
\hline $\begin{array}{lr}\text { Duy } & \text { Ngoc } \\
\text { Pham } & \text { et } \\
\text { al.[12] } & \end{array}$ & $\begin{array}{l}\text { AODV } \\
\& \text { DSR }\end{array}$ & Mentioned & $\begin{array}{l}\text { Energy Consumption, } \\
\text { Routing Overheads, Hop } \\
\text { Count, Packet Delivery } \\
\text { Ratio. }\end{array}$ & No of Nodes \\
\hline $\begin{array}{l}\text { Sunil Taneja } \\
\text { et al.[13] }\end{array}$ & AODV & $\mathrm{NS} 2[18]$ & $\begin{array}{l}\text { Packet Delivery Ratio, } \\
\text { Exhausted Nodes. }\end{array}$ & $\begin{array}{l}\text { Pause time, Speed, } \\
\text { No of Sources. }\end{array}$ \\
\hline
\end{tabular}

\section{PROPOSED ALgORITHM}

Here the aim is to reduce the energy consumption, of the routing protocol so that network lifetime gets improved. Many other researchers have also worked in this direction, the main advantage of this work over the previous works is that by using this approach the network routing overhead and number of exhausted nodes gets decreased. As well as, no node dies in the network due to battery power because always the path with nodes having sufficient battery power has been chosen. 
In proposed algorithm, the route selection depends upon the battery levels of nodes which are divided in to three states that are dead state (Battery less than 20\%), critical state (Battery less than 50\% and greater than 20\%) and active state (Battery greater than 50\%). To find an Energy Efficient route, a Route REQuest (RREQ) packet is broadcasted in the network. The RREQ packet can be processed by either the destination node or the intermediate node. The RREQ packet contains a source ID, the link energy information for each link in the route. The neighboring nodes do the rebroadcasting of RREQ packet only if their battery status is greater than the selected threshold level i.e. critical state and lies in active state. Otherwise, the RREQ packet would be queued. When the RREQ packets reaches to the intended destination, the destination node computes the total energy of route and the Rfactor of received requests. Rfactor is defined as follows

$$
\text { Rfactor }=\frac{\text { Total route energy }}{\text { Number of hopes in that route }}
$$

Whereas the Total route energy is the sum of remaining battery power of all nodes in that route. Then sends the route reply (RREP) through the route having maximum value of Rfactor and selects a route which is energy efficient. The calculated routes total energy is the maximum energy of a link for that route.

Our approach is described as follows

Step1: The nodes which are not participating in the route computation process are in sleep mode from the start.

Step2: Source node S broadcasts a route-request (RREQ) packet to its neighboring nodes.

Step3: The neighboring nodes do the rebroadcasting of RREQ packet only if their battery status is greater than the selected threshold level. Otherwise, the RREQ packet would be queued.

Step4: When the RREQ packet reaches to the intended destination, destination selects a route which is energy efficient (according to the defined formula). Then it constructs a route-reply (RREP) packet and sends it back to the source node.

\section{Simulation \& Performance Results}

To analyze the performance of proposed approach, NS2 [18] simulator has been used. The simulator comes with a number of stand-alone programs which generate simulation traffic. The agents are also attached to the traffic according to the user's requirements. After defining all the parameters, simulation has been started \& the output is compared with standardized AODV (Adhoc On-demand Distance Vector) routing protocol [23] in terms of various performance metrics like Energy Consumption, Packet Delivery Ratio, Network Life Time, Network Routing Overhead and number of exhausted nodes. A number of scenarios has been generated by varying number of nodes, speed and pause time. The scenario model for examining the routing protocol performance is given below in Table 2 . 
Table 2: Simulation Parameters

\begin{tabular}{|c|c|}
\hline Parameters & $\begin{array}{c}\text { Variation of } \\
\text { Parameters }\end{array}$ \\
\hline Area(m x m) & $1000 \times 1000$ \\
\hline Nodes & $10,30,50,70,90,100$ \\
\hline Simulation Time(s) & $500 \mathrm{sec}$ \\
\hline Node Speed(m/s) & $1,3,5,7,9,10$ \\
\hline Pause Time(s) & $10,15,20,25,50,100$ \\
\hline Traffic Type & FTP,CBR \\
\hline Packet Size & 512 \\
\hline Protocol Used & AODV \\
\hline
\end{tabular}

Different types of networks exist, and the traffic pattern of each network differs from that of the other network. Furthermore, the traffic pattern for a simulation should resemble an actual MANET's traffic. Most networks favor certain applications and most network applications favor the use of a specific transport protocol. For example, the majority of traffic on one network may use TCP while another network will primarily use UDP. Here we use both UDP and TCP traffic flows.

Our algorithm is named as New AODV and we have compared its performance with AODV routing protocol.

\subsection{Simulation results on the basis of packet delivery ratio and network routing overheads:-}

Packet Delivery Ratio (PDR) is the ratio of total number of packets successfully received by the destination node to the number of packets sent by the source node.

$$
P D R=\frac{\text { Number of packet received }}{\text { Number of packet sent }}
$$

Network Routing Overhead (NRO) is defined as the ratio of the number of routing packets sent over a single data packet in the network.

Figure 1 depicts the packet delivery ratio by varying pause time as a parameter for AODV and New AODV protocol. Whereas, figure 2 depicts the Network Routing Overhead of AODV and New AODV. The results are on the basis of 50 mobile nodes having TCP connections. Pause time has been varied from 10 seconds to 100 seconds. 
The results show that, the new AODV starts outperforming the original AODV in terms of packet delivery ratio and Network Routing Overhead. Because we send less number of route reply (RREP) packets for a route selection which results in decreased routing overhead.

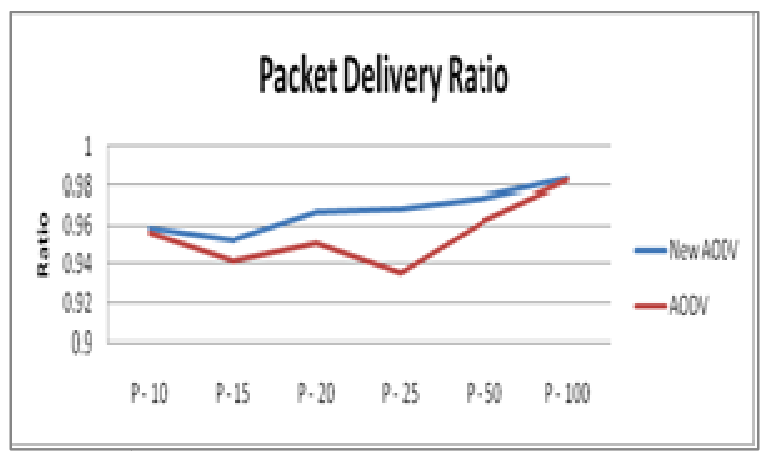

Figure 1: Packet delivery ratio by varying pause time.

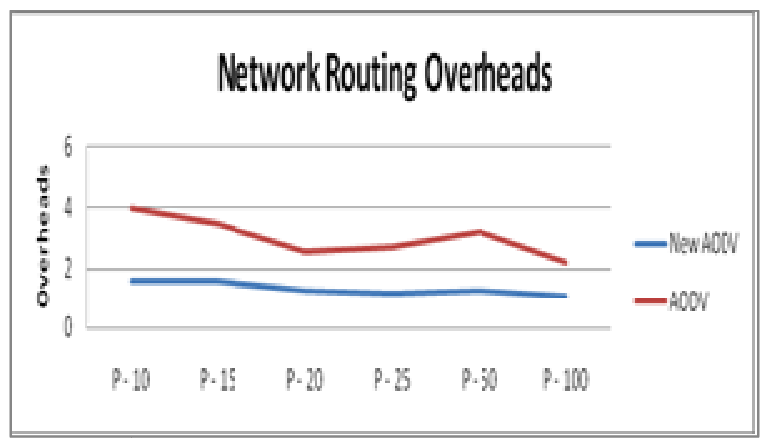

Figure 2: Network Routing Overhead by varying pause time.

By varying speed various scenarios are generated and the performance of AODV and new AODV in terms of packet delivery ratio and network routing overhead is illustrated in figure 3 and 4 respectively. The results are on the basis of 50 mobile nodes having TCP connections. Speed has been varied from $1 \mathrm{~m} / \mathrm{s}$ to $10 \mathrm{~m} / \mathrm{s}$. The results show that, the new AODV outperforms the AODV protocol irrespective of speed. As the node speed increases network routing overhead gets increased because of the mobility of nodes topology changes frequently. So packet delivery ratio decreased but in comparison to AODV, New AODV has better performance.

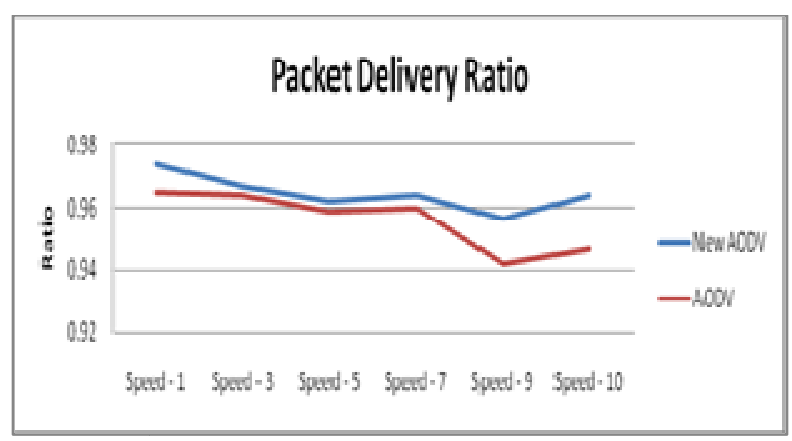

Figure 3: Packet delivery ratio by varying speed of nodes 


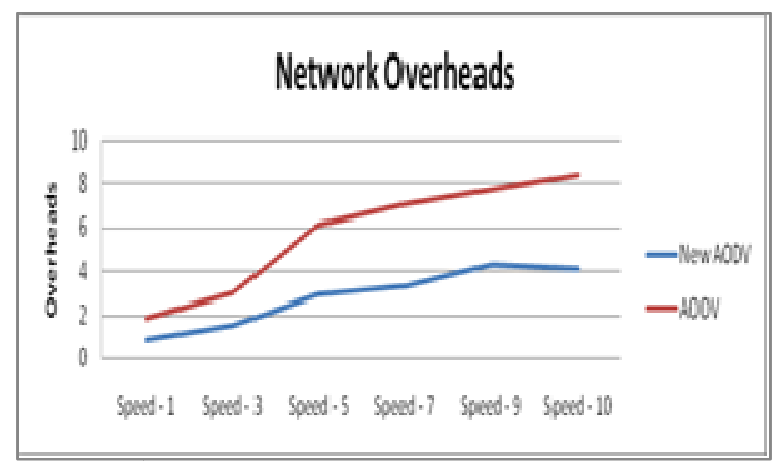

Figure 4: Network Routing Overhead by varying speed of nodes

\subsection{Simulation results on the basis of exhausted nodes and dead nodes:-}

Exhausted nodes are the number of nodes that contains the energy less than 30 percent at the end of each simulation run and the total number of nodes died due to their total energy supplied to them is consumed, and at the end of the simulation these nodes are called dead nodes.

Network Lifetime (NL) is defined as the time taken for certain amount of the nodes in a network to die.

The figure 5 and 6 illustrates the behavior of AODV and new AODV after varying the total number of nodes with respect to exhausted nodes and Network Lifetime. It can be observed that on an average, more number of nodes die till the end of simulation in case of AODV while no dead nodes were found in case of New AODV and same is for the case of exhausted nodes. This is due to the fact that New AODV routes have to be selected not only to deliver packet with minimum energy consumption but also to distribute the load based on the battery threshold levels and the battery level threshold never let the node die in the network.

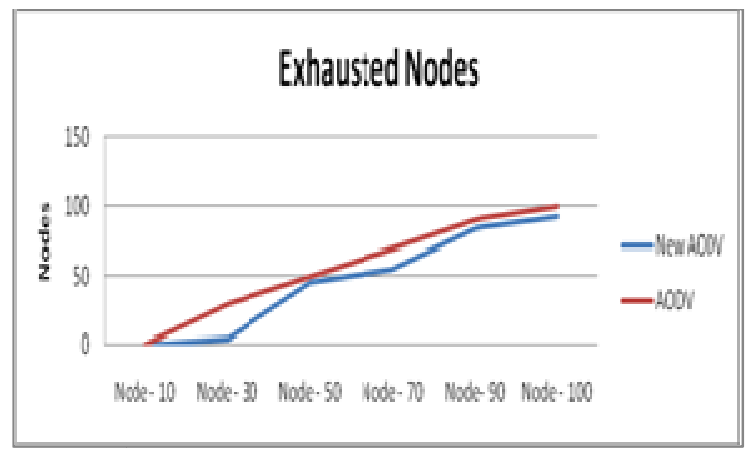

Figure 5: Number of exhausted nodes by varying nodes 


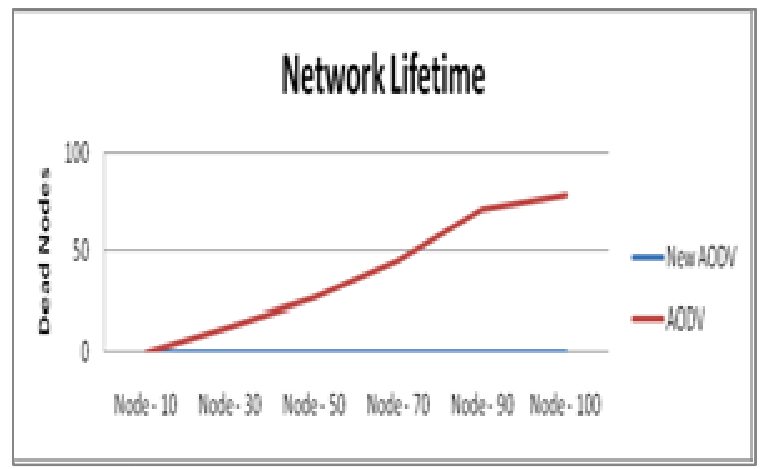

Figure 6: Number of dead nodes by varying nodes

\section{CONCLUSION}

A lot of work has been done in the field of power aware routing over mobile ad-hoc networks. Their research work has been considered and an effort has been made to modify the existing AODV protocol by applying battery thresholds and energy efficient route calculation formula. This causes a large decrement in network routing overheads and exhausted nodes. The proposed algorithm minimizes the energy consumption of a node to transfer the data from source to destination, so network lifetime gets increased. The proposed algorithm makes optimum utilization of battery of a node during route selection. The performance of proposed routing protocol is analyzed on the basis of Energy Consumption, Packet Delivery Ratio, Network Life Time, Network Routing Overhead and number of Exhausted nodes. Simulation results indicate that the proposed scheme consumes less energy in the network and Network Routing Overhead get decreased. Results are better in denser medium as more nodes are available at the time of route selection. This Scheme gives best results when TCP traffic is used and the nodes are moving at a high speed. At last, it can be said that by looking at the battery levels of the nodes, performance of the routing protocol is improved and the network lifetime gets increased.

\section{REFERENCES}

[1] Sunil Taneja and Ashwani Kush, (2010)“A Survey of Routing Protocols in Mobile Ad Hoc Networks" International Journal of Innovation, Management and Technology, Vol. 1, No.3, August.

[2] G.Vijaya Kumar, Y.Vasudeva Reddyr , Dr.M.Nagendra (2010) "Current Research Work on Routing Protocols for MANET: A Literature Survey" International Journal on Computer Science and Engineering, Vol. 02, No. 03, 706-713.

[3] Sandip Vijay, S. C. Sharma, "EEN: An Energy Efficient Multi-hop Ad Hoc Wireless Networks" First International Conference on Emerging Trends in Engineering and Technology.

[4] Shwani Kush, Sunil Taneja and Divya Sharma, (2010) "Energy Efficient Routing for MANET" International Conference on Methods and Models in Computer Science (ICM2CS-2010).

[5] S.Preethi, B. Ramachandran, (2011) "Energy Efficient Routing Protocols for Mobile AdHoc Networks" IEEE.

[6] G. Varaprasad, (2010) "Lifetime enhancement routing algorithm" Published in IET Communications.

[7] Kwan-Woong Kim1, Jeong-Soo Lee2, Kyoung-Jun Hwang1, Yong-Kab Kim1, Mike M.O. Lee3, Kyung-Taek Chung4, and Byoung-Sil Chon5, (2006) "LRCA: Enhanced Energy-Aware Routing Protocol in MANETs" AI 2006, LNAI 4304, pp. 897 - 901. 
[8] X. Xiang, X. Wang, and Y. Yang, (2011) "Supporting Efficient and Scalable Multicasting over Mobile Ad Hoc Networks" IEEE Transactions On Mobile Computing, Vol. 10, No. 5.

[9] Nivedita N. Joshi1 and Radhika D. Joshi, (2011) "Variable Range Energy Efficient Location Aided Routing for MANET" CCSEA 2011, CS \& IT 02, pp. 321-333.

[10] Dheeraj Kumar Anand and Shiva Prakash, (2010) "A Short survey of Energy-Efficient Routing Protocol for Mobile Ad-Hoc Networks" International Conference on Advances in Recent Technologies in Communication and Computing.

[11] Ll Bing, JIN Zhigang and SHU Yanati, "Cross-Layer Design of Energy-Saving AODV Routing Protocol" School of Computer Science and Technology, Tianjin University, Tianjin 300072, China.

[12] Ngoc Duy Pham and Hyunseung Choo, (2008) “Energy Efficient Ring Search for Route Discovery in MANETS" IEEE Communications Society subject matter experts for publication in the ICC proceedings.

[13] Sunil Taneja, Ashwani Kush, Amandeep Makkar and Bharat Bhushan, (2011) "Power Management in Mobile Adhoc Network" International Transaction Journal of Engineering, Management, \& International Transaction Journal of Engineering, Management, \& Applied Sciences \& Technologies.

[14] Ramesh Karri And Piyush Mishra, "Modeling Energy Efficient Secure Wireless Networks Using Network Simulation" Polytechnic University, Brooklyn.

[15] K.Srinivas, A.Nagaraju, S.Ramachandram and G.Narsimha, (2010) "Performance Evaluation of Routing Protocols in Static and Dynamic Ad-hoc Networks based on Energy Consumption" Second Vaagdevi International Conference on Information Technology for Real World Problems.

[16] GloMoSim User Manual, http://pcl.cs.ucla.edu/projects/glomosim.

[17] D.N.Pham,V.D.Nguyen, V.T.Pham, N.T.Nguyen, X.BacD, T.D.Nguyen, C.Kuperschmidt and T.Kaiser, (2010) "An Expending Ring Search Algorithm For Mobile Adhoc Networks", International Conference on Advanced Technologies for communication, Vietnam.

[18] NS-2, http://www.isi.edu/nsnam/ns/doc/index.html [Accessed August 11, 2007].

[19] Kuo-Qin Yan, Shu-Ching Wang, Mao-Lun Chiang and Lin-Yu Tseng , (2009) "A Fuzzy-based Power-aware Management for Mobile Ad hoc Networks" ACM Digital Library, Journal Computer Standards \& Interfaces, Volume 31, Issue 1.

[20] Mohammed Tarique and Rumana Islam, (2007) "Minimum Energy Dynamic Source Routing Protocol for Mobile Ad Hoc Networks" IJCSNS International Journal of computer Science and Network Security, VOL 7 No. 11.

[21] M. Maleki, K. Dantu and M. Pedram, (2002) "Power-aware Source Routing in mobile ad hoc networks" Proceedings of ISLPED' Monterey,CA.

[22] Kevin Fall, Kannan Varadhan, (2008) "The NS Manual".

[23] Geetha Jayakumar, G. Gopinath, "AdHoc Mobile Wireless Networks Routing Protocols - A Review" Journal of Computer Science 3 (8): 574-582, 2007ISSN 1549-3636 


\section{Authors}

Pallavi Kaliyar She received her B.E in Computer Science and Engineering from Jaipur Engineering College \& Research Center, Jaipur in 2008, M.Tech from jaypee Institute of Information Technology, Noida in 2012. She is currently working as an Assitant Professor in Shri Ram College of Engineering \& Technology, Muzaffarnagar. Her research interests include Mobile Adhoc Networks, Sensor networks and Network Security.

Kavita Pandey She did B.Tech in Computer Science from M.D. University in year 2002 and M.Tech. in Computer Science from Banasthali Vidyapith University in year 2003. She is pursuing PhD from JIIT,Noida. She is working as a Senior Lecturer in JIIT,Noida. Her current research interests include Adhoc Networks, Optimization Techniques and Network Security.

Gagan Singla M.Tech working as an Assistant Professor in the Department of Computer Science in Aryabhatta Group of institutions, Barnala, Punjab, India. His research interest lies in the area of Mobile Adhoc Networks and Network Security.
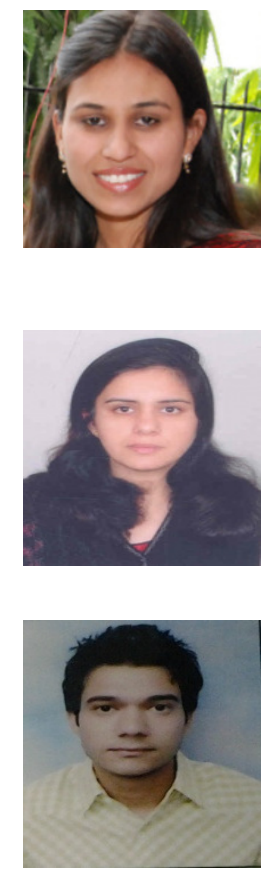